\title{
SUR LA SIGNIFICATION DES NOMS PROPRES DANS LES ROMANS DE CHRÉTIEN DE TROYES
}

\author{
Marcela-Cristina OȚoiU \\ Universitatea Tehnică din Cluj-Napoca, \\ Centrul Universitar Nord din Baia Mare, România
}

\section{On the meaning of characters' names in the writings of Chrétien de Troyes}

\begin{abstract}
The present article aims to analyze the relationship that the characters in the writings of Chrétien de Troyes have with their names. One particular trait of this French writer, commonly regarded as the father of chivalry, is the use of heroes whose names echo their physical and moral features, anticipate their deeds and foreshadow their destinies. It is by means of an etymological probing of these names that we can outline a typology of these characters. These heroes' names occur at different places in the narrative: at the beginning, midway, or at the end. The latter situation, when the hero's name is revealed at the very end, is a peculiar feature in the writings of Chrétien de Troyes. An intrinsic part of the art of characterization, the choice of heroes' names helps the reader get a fuller grasp of the narrative's moral stakes.
\end{abstract}

Keywords: proper names, etymology, message, destiny, Round Table legends.

Chrétien de Troyes est le maître incontestable du roman courtois du XIIe siècle et le père du roman europééen, dont la création ne cesse de fasciner encore bon nombre de lecteurs et de chercheurs. L'attraction pour les légendes arthuriennes constitue un centre d'intérêt constant, grâce à leur poids d'aventure, de merveilleux et de mystère celtique qui côtoie le mythe.

Bien que les personnages appartiennent à un temps mythique indéfini, leur comportement et leur idéologie reflètent les courants de pensée du XIIe siècle français, c'est-à-dire l'idéologie courtoise contemporaine de l'écrivain. L'effort de Chrétien de Troyes se dirige vers l'adaptation des anciennes légendes qui circulaient oralement au gré de la littérature courtoise de son temps.

Les noms propres ont un poids majeur dans l'oeuvre de Chrétien de Troyes. Ces noms, qui sont pour la plupart d'origine celtique, sont empruntés généralement à la matière de Bretagne, principalement à l'Historia Regum Britanniae de Geoffroy de Monmouth (vers 1100 - 1155). Chrétien de Troyes met en scène les noms de ses personnages dès le titre même de ses romans. En ce qui concerne les titres des romans que l'on a conservés de Chrétien de Troyes, on peut observer que, dans quatre cas sur cinq, ils contiennent un nom propre suivi d'un ajout explicatif, sauf dans le cas du premier roman, Érec et Énide: 
Erec et Enide - titre composé d'un nom masculin et d'un nom féminin, qui suggère l'histoire d'amour d'un couple où l'on accorde une égale importance aux deux partenaires.

Cligès ou la fausse morte - titre qui propose une possibilité d'opter pour une variante ou pour une autre. Mais, tandis On note également que la première partie du titre contient un nom masculin, Cligès, et que la deuxième partie renvoie à une référence féminine.

Dans le cas des romans Lancelot ou Le chevalier de/à la charrette et Yvain ou Le chevalier au lion, les deux titres contiennent une périphrase qui constitue un indice de la future action et qui oriente en même temps le sens de la lecture. La corrélation entre le nom propre et l'ajout explicatif n'apparaît qu'à partir du contenu du texte du récit, n'étant pas valable dans d'autres contextes. Lancelot n'a le nom de Chevalier de la charrette que dans le roman de Chrétien de Troyes. Ici, il monte dans la charrette des condamnés afin d'arriver plus vite à destination, près de la reine qu'il aime.

Le titre Yvain ou Le Chevalier au lion propose une option pour une autre désignation du héros, qui n'est plus péjorative comme dans le cas du Chevalier à la charrette. Le lion, compagnon noble et sauvage du héros, l'enrichit aussi de sa symbolique solaire.

Perceval ou le Conte du Graal nous offre par son titre une invitation à suivre une histoire de formation et d'aventure, à forte symbolique chrétienne. Il n'y a pas d'équivalence entre les deux termes, car il s'agit de l'intégration de l'histoire personnelle de Perceval dans l'un des contes du Graal, d'autant plus que Perceval ne réussit pas à en déchiffrer le mystère. D'ailleurs, l'histoire de Perceval n'est pas la seule à être racontée dans ce roman resté inachevé, mais, à côté de celle-ci, s'ouvre l'histoire parallèle de Gauvin, toujours destinée à l'échec.

\section{Signifié et signifiant des noms propres chez Chrétien de Troyes}

Les noms propres englobent, pour la plupart, les qualités des héros, qui sont visibles dans l'étymologie même. Nomen est omen - c'est-à-dire le nom est presage - peut être valable dans le cas de bien des personnages présents dans les romans de Chrétien de Troyes. Comme le soulignent Henry et Renée Kahane, „l'étymologie d'un nom, surtout dans la littérature médiévale, peut ouvrir la voie, sur le plan synchronique, à l'explication typologique d'un personnage littéraire, ou, sur le plan diachronique, éclairer la genèse intellectuelle de l'oeuvre où ce nom figure" (1961: 113). Autrement dit, dans les mots de Claude Buridant, les noms propres de la littérature médiévale ont „une dénomination juste, motivée, dans laquelle on peut découvrir une véritable signification que révèle la procédure étymologique”, raison pour laquelle „'étymologie du nom propre devient alors partie intégrante du portrait" (Buridant 1998: 20).

Selon Gérard Genette, qui parle de la fonction de désignation des noms propres, on peut découvrir une „procédure étymologique” qui consiste en „un accord de convenance entre désignation et signification (entre désigné et signifié), la seconde venant en quelque sorte redoubler, conforter, confirmer la première: en un mot, la motiver, en lui donnant un sens" (Genette 1976: 24). 
Ainsi, le nom propre peut préfigurer ou rétrofigurer la personnalité, le caractère, le destin, en tant que „programme” et „clé de son destin” (Buridant 1990: 50).

L'explication étymologique des noms a attiré l'attention des chercheurs dans leur effort de comprendre l'origine mythique des personnages. Pour Philippe Walter, l'auteur du Dictionnaire de mythologie arthurienne, le nom peut être un véritable „pilier du mythe". On peut ainsi établir une corrélation entre le nom propre et le caractère d'un même personnage, ce qui mène à une meilleure compréhension du texte. Analysons donc les principaux noms propres qui apparaissent dans les romans de Chrétien de Troyes et leur signification.

En ce qui concerne le nom d'Arthur, on a formulé plusieurs hypothèses, dont nous énumérons trois des plus importantes:

Selon une première hypothèse, son origine serait le nom latin Artorius, nom d'un officier romain attesté en Angleterre au milieu du Vle siècle.

Une deuxième hypothèse voit le nom provenir du gallois arthos, „ours”. On peut rapprocher ce nom de celui de la déesse ourse celtique Artio.

Une autre hypothese est celle de la provenance de ce nom de l'étoile Arcturus, l'une des plus brillantes étoiles de l'hémisphère nordique, qui se trouve non loin de la Grande Ourse et de la Petite Ourse. On peut remarquer aisément dans ce cas autant la proximité physique que lexicale (Arktoûros signifie le gardien des ours).

En effet, Arthur réunit les qualités de l'ours, animal totémique, remarquable par sa force, sa résistance, sa stabilité. Chez les Celtes, l'ours est devenu l'emblème de la royauté avant celle du lion qui s'imposera au XIIe siècle. Le roi-ours Arthur (Artu en ancien français) est la figure mythique, le symbole d'un état féodal idéal, qui rassemble autour de lui une confrérie souveraine de guerriers, les chevaliers de la Table Ronde (Walter 2003: 41). Walter reprend l'affirmation de René Guénon pour lequel le nom d'Arthur est mis en relation avec le symbolisme polaire et en donne une explication: „tout comme la Grande Ourse indique le pôle géographique, l'ours-roi Arthur est le pôle autour duquel s'organise la Table Ronde" (48).

Arthur est le roi mythique des Bretons qui, au VIe siècle, avait résisté à l'invasion saxone. Son nom se trouve mentionné pour la première fois chez Nennius, au IXe siècle, dans l'Histoire des Bretons Arthur y apparaît non pas comme un roi, mais comme un chef guerrier qui aurait remporté plusieurs victoires dans la lutte contre les Saxons.

Quand même, l'existence réelle d'Arthur reste encore incertaine. Son nom n'est pas mentionné dans d'autres textes (Gildas au VIe siècle ou Bède au VIIIe siècle). Chez Geoffroy de Monmouth, dans Historia Regum Britanniae, histoire tout à fait imaginaire, écrite vers 1135, la figure d'Arthur a pris du contour et de l'importance, devenant source d'inspiration pour toutes les légendes écrites plus tard (pour Wace par exemple, qui écrit en 1155 le Roman de Brut).

Guenièvre (du gallois Gwenhwyfar ou Gwenhwyar) signifie «blanc fantôme » ou blonde fée. Cela est en relation avec ses caractéristiques physiques (elle a le teint très blanc et les cheveux d'or qui rappellent le blanc fantôme des sagas nordiques), car elle a 
vraiment une nature féerique. Par sa beauté, elle ressemble à une fée qui évoque l'Autre monde. On observe un caractère d'amiguité dans l'appellation blanc fantôme - a la fois visible et irréelle, fantasme à laquelle aspire son amoureux. Guenièvre est l'incarnation même de la dame courtoise; belle et spirituelle, elle a une emprise totale sur Lancelot, le domine par son pouvoir et sa volonté. Son statut privilégié, de femme idéale, ne l'empêche pas de manifester une certaine ambiguiité au niveau du comportement.

Pour l'étymologie du nom de Lancelot il y a aussi plusieurs hypothèses:

Selon R. Bromwich, l'étymologie de ce nom reste totalement inconnue, car aucune explication n'est satisfaisante (voir Walter 2003: 43). Selon d'autres hypothèses, le nom Lancelot est dérivé soit d'un terme germanique (de lanzo, terre), soit, selon Ferdinand Lot, du gallois, (Llenlleauwe ou Llenvlaic), soit, le plus probablement, il est un dérivé du latin ancellus, serviteur, ou du normand ancel, ayant la même signification.

En vieux français, lancel signifiait la même chose, serviteur. En effet, il est le serviteur parfaitement dévoué de la reine, à laquelle il satisfait sans hésiter tous les caprices.

Quand à son nom complet, Lancelot du Lac, (il est présenté ainsi par l'une des demoiselles de la reine) on peut penser aux valences symboliques de l'eau dont il provient: fils du roi Ban de Bénoïc et de la reine Élaine, il avait été enlevé par Viviane, la Dame du Lac et élevé par celle-ci au fond de l'eau. Ses qualités naturelles sont agrandies par la force élémentaire de l'eau (Zimmer 1994: 134-135) car il porte, sans doute, „la magie du Lac” et la force vitale de l'eau. Ainsi s'expliquerait son côté irrationnel, instinctuel, sa passion démesurée pour la femme d'Arthur. Par sa sensualité doublée de courtoisie il est considéré comme le plus attractif et le plus splendide chevalier de la Table Ronde, même s'il trahit la confiance de son roi. Incarnant l'idéal masculin des femmes, „l'archétype animus”, son psychique est nettement marqué par „le lac de l'inconscient" (Zimmer 1994: 157), tant il est perdu dans l'adoration permanente de la reine.

Erec - dérivé du scandinave Eric, dont le premier morphème ei signifie seul, et le second morphème signifie roi (rich). Le sens global serait de chef unique. En effet, Erec est parmi les premiers chevaliers, il possède des qualités uniques et exemplaires. Une autre théorie de l'origine de ce nom est avancée par Jean-Pierre Foucher, qui voit dans l'épopée galloise de Ghereint l'origine du nom Erec, après avoir passé par la variante Guerec. D’autre part, Érec est, selon le mème chercheur, le nom d'un chef de guerre vannetais du haut Moyen Age (le pays de Vannes s'appelait en breton le „Bro-Erech”, c'est-à-dire „le pays d'Érec”. Le nom d'Énide viendrait lui aussi de la même zone géographique: Gwened, „la blanche”, forme rapprochée de celle du nom d'Énide, est le nom breton de la ville de Vannes (Foucher 2011:34-35).

Gauvin est le neveu d'Arthur et le meilleur chevalier de la Table Ronde, dont le nom signifie faucon. C'est le cas d'un chevalier idéal, parfaitement dévoué, dont le nom est mentionné dans presque toutes les legendes arthuriennes. L'étymologie de son nom reste encore discutable (Walter 2003: 43).

Une situation particulière est celle du nom Keu, le plus court nom de chevalier et celui qui a le plus grand nombre de variantes écrites: Cai, Cei, Kay, Kei, Key, Keu, 
Ké auxquels s'ajoutent Queuz, Kex et Kés en vieux français). C'est le nom du sénéchal d'Arthur, qui est en même temps son frère nourricier. Le nom, dérivé du latin Caius, a été plus tard rapproché de celui de key en anglais, qui signifie clé. Grâce a sa ressemblance avec son homophone key, il sera associé plus tard avec cet objet qui deviendra son emblème dans les livres d'armoiries.

Pour le nom du roi Baudemagus on n'a pas d'étymologie exacte, mais on pense à sa connotation positive en raison de son rapprochement de l'adjectif beau placé à côté de mage - qui vient de magus, homme sage et instruit, pratiquant la magie ou l'astrologie. En effet, le roi Baudemagus a un beau caractère, il est juste et sage, soutient la paix et condamne les méchancetés de son fils Méléagant.

Par contre, le nom de Méléagant a des connotations nettement négatives. Il provient du nom du dieu celte Melwas, Seigneur du Pays de l'Eté, identifié aussi au Royaume de l'Autre monde. De par son nom, Méléagant est possesseur de caractéristiques infernales, qu'il ne cesse de montrer sur tout le parcours du récit. On n'est pas surpris que ce roi, qui enlève la reine et l'emporte dans son royaume, est cruel, excessif, violent et arrogant. Il est l'opposant de Lancelot qui n’a d'autre désir que de sauver la reine des mains de son ravisseur.

Soredamor - nom composé de sor - soleil, or ou blonde et amor; la signification du nom devient blonde ou dorée d'amour (par extension rayonnante ou resplendissante d'amour). L'explication de ce nom nous parvient au moment où, consumée par l'amour, la jeune fille trouve elle-même la raison de son nom:

\begin{abstract}
Amour ne me donne que de bonnes leçons: ce n'est pas sans raison que que je suis appelée Soredamor, c'est dire: Blonde d’Amour. Je dois aimer et être aimée. Je le dois prouver par mon nom car en ce nom je ne trouve qu'Amour. Si la première partie de mon nom est couleur d'or c'est que les meilleurs sont les plus blonds. Pour cela je tiens mon nom pour le plus beau car il est de la couleur qui s'accorde avec tout ce qui est le meilleur. Qui m’appelle par mon vrai nom toujours me rappelle l'amour. Une moitié de mon nom dore l'autre moitié d'une dorure claire et blonde et l'on dit Soredamor comme on dirait „Surdorée d'Amour” (Chrétien de Troyes 2011: 106).
\end{abstract}

Blanchefleur, le nom de la jeune fille aimée de Perceval, est un nom composé qui suggère la beauté féminine, la grâce et la pureté. La jeune fille qui porte ce nom l'illustre totalement par son apparence physique et par ses qualités spirituelles.

Dans Cligès, roman qui a une double origine, celtique et orientale-byzantine, les noms ne gardent plus l'étymologie celtique à laquelle on était habitué dans les autres romans de Chrétien. Ainsi, on a identifié l'origine du nom de Cligès dans le nom du sultan d'Iconium, Kilidj Arslan II (1156-1192). Quant au nom de Fénice, on voit que celui-ci est dérivé du latin phoenix, nom de l'oiseau fabuleux qui meurt et renaît de sa propre cendre. Le nom est pleinement significatif pour le destin de la jeune femme Fénice qui aura une fausse mort causée par un philtre, et reviendra ensuite à la vie pour connaitre un amour accompli.

Thessala, la nourrice de Fénice, est appelée ainsi, explique Chrétien, parce qu'elle 
était originaire de Thessalie, pays où „les charmes diaboliques sont enseignés et pratiqués” et où les femmes sont „expertes en envoûtements et sortilèges” (Chrétien de Troyes 2011: 118).

Yvain, appelé aussi Owein en gallois et Ewain en anglais, du celte $y v$-if, arbre sacré de la religion des Celtes, est le héros du roman éponyme Yvain ou le chevalier au lion. Si le sens étymologique du nom n'est pas trop évident, le fait d'être le chevalier au lion le distingue de tous les autres héros. Après avoir été plusieurs fois aidé par le lion, Yvain, en signe de reconnaissance, a pris le nom de Chevalier au lion. C'est au moment de la rencontre avec sa dame, qui ne le reconnait pas, qu'il se donne cette nouvelle identité.

Perceval, héros du roman éponyme, a un nom à étymologie incertaine. Selon quelques-uns ce serait un prénom apparenté à Pierre, selon d'autres il serait d'origine galloise: Perredur était mentionné parmi les chevaliers de la Table Ronde et son nom aurait été repris et francisé par Chrétien de Troyes.

Le nom du Roi Pêcheur, bien que motivé par la condition du personnage (ayant les pieds malades, il ne peut se déplacer qu'en barque où il passe son temps à pêcher), a aussi une symbolique religieuse, le poisson étant un ancien symbole chrétien. Ce n'est pas par hasard qu'il se trouve dans la proximité du Graal et qu'il ne peut guérir qu'au moment où son mystère sera révélé.

\section{Présence ou absence des noms propres}

Tandis que les noms des héros de premier plan sont mentionnés bien des fois, beaucoup de personnages de Chrétien de Troyes restent anonymes. On peut remarquer l'absence surtout des noms des personnages féminins, qui ne sont désignés que par leurs traits individuels les plus poignants (ex. la demoiselle entreprenante, la dame veuve, la demoiselle Sauvage, la demoiselle à la mule, la demoiselle qui a ri, la demoiselle méchante, la Demoiselle aux Manches Petites).

Parmi les femmes qui ne sont pas d'origine noble, seule Lunette, la servante de Laudine, la femme d'Yvain, est appelée par son nom. Ce n'est pas d'ailleurs par hasard, parce que l'on peut penser, quant à Laudine, à la figure de Diane chasseresse et en même temps deesse de la Lune. Tout comme Énide, la femme d'Érec, Laudine n'est appelée par son nom qu'au moment de son mariage avec Yvain.

Même le nom de la reine Guenièvre n'est mentionné que deux fois dans Lancelot ou le chevalier à la charrette, l'appelatif de reine étant choisi pour suggérer au mieux son statut tout puissant, au-dessus du chevalier qui l'aime.

Le nom du protagoniste du roman peut s'absenter jusqu'à un certain moment de l'action. C'est le cas d'Énide, dont on ne connaît pas le nom jusqu'au moment du mariage, malgré le fait qu'elle était „la plus belle fille et la plus sage” des jeunes filles de la cour.

L'existence des sobriquets pour Lancelot, Yvain et Perceval montre l'importance du regard extérieur de la collectivité. L'homme est toujours vu et apprécié dans des termes visant les qualités ou les défauts comme bon, mauvais, fier, courageux, orgueilleux, couard, cruel, etc. 
En effet, Lancelot construit son identité sous le regard d'autrui. Tout premièrement, c'est le regard de la reine qui lui dicte les actions et le comportement au combat. Selon la volonté de la reine, Lancelot lutte au mieux ou au pire, ce qui lui attire des appréciations opposées. Ensuite c'est le regard de la collectivité qui construit l'identité du personnage: celle-ci le voit comme preux ou méprisable en fonction de la situation où il se trouve. Lancelot change de nom toutes les fois qu'il passe par une épreuve humiliante ou par une épreuve qui lui permet de se mettre en valeur. $\mathrm{Si}$, au début du roman, il n'est pas désigné par son nom, on apprend le nom du personnage au moment où il se fait remarquer par ses actions qui l'individualisent. L'intention de Chrétien est de montrer que le personnage mérite son nom légendaire (le public de Chrétien connaissait sans doute l'histoire de Lancelot et de Guenièvre qui circulait à l'époque, tout comme d'autres légendes du cycle arthurien).

Arrivé au comble du mépris, Lancelot cesse d'avoir un nom, car les gens ne veulent plus le prononcer. Appelé au début le chevalier de la charrette, il se voit insulté et subit une grande offense mais peu lui chaut. Plus tard, il est appelé libérateur, sauveur des prisoniers du royaume de Gorre, ce qui changera son nom en renom. C'est d'ailleurs vers ce renom qu'évolue le personnage, qui ne se contente pas de son simple nom.

La révélation du nom de Perceval constitue un moment à part, empli d'importance et de solennité. Si pendant une bonne partie du roman on ne connait pas le nom du protagoniste, qui est désigné seulement par l'appellatif garçon, le dévoilement de son nom se fait selon une technique du suspense. Le nom qui manquait, tant attendu, obtient ainsi un autre poids:

Et lui, qui son nom ne savait, soudain le connut et lui dit que c'est Perceval le Gallois. Mais il ne sait s'il dit vrai ou non. Il dit vrai, pourtant ne savait (Chrétien de Troyes 2004: 99).

Cette façon de voiler et de dévoiler le nom du personnage constitue l'emblème stylistique de Chrétien. À la différence des chansons de geste où il y a de longues énumérations de noms, Chrétien joue avec l'identité du personnage, qu'il aime cacher, pour la révéler ensuite cérémonieusement à un moment significatif du récit.

Dans les romans de Chrétien de Troyes où il y a beaucoup de figurants qui restent anonymes, où même les personnages les plus importants ne sont nommés que beaucoup après le commencement de l'action, quelquefois seulement à la fin, on est d'autant plus étonné d'apprendre le nom de quelques personnages accessoires. On apprend ainsi le nom d'un serf de Cligès, Jean - qui semble avoir été choisi pour représenter le nom masculin le plus usuel, et le nom de Trébuchet le forgeron, détenteur d'un savoir presque magique de réparer les armes abîmées. À noter qu'en ancien français, un „trébuchet” pouvait avoir plusieurs significations. Parmi celles-ci, celle qui a pu correspondre le mieux à l'origine du nom de ce personnage est sans doute de „réparateur de machines de guerre" (Greimas 1970: 639).

Esclados le Roux, le gardien de la fontaine et le mari de Laudine, dont nous 
apprenons le nom seulement quand il est mort, est un personnage qui appartient aussi à l'Autre monde celtique. En plus, sa rousseur est un indice de son altérité (Vișan 2003: 61).

\section{Particularités des noms propres}

1. Les diminutifs des noms sont peu présents dans ces récits. On doit remarquer dans Cligès l'apparition des noms de deux frères, Alexandre et Alis, dont l'aîné porte le nom d'un illustre prédecesseur, tandis que le cadet n'a qu'un nom sans réverbérations. Malgé le fait qu'Alis était au Moyen Âge un prénom courant, d'origine germanique parfaitement attestée, tout comme ses nombreuses variantes: Alais, Allais, Alès, Allès, Alix, A LIPS, on pourrait le prendre, au point de vue phonétique seulement, pour une variante réduite du nom d'Alexandre, ressemblant ainsi à un diminutif. En plus, on peut aussi constater sa ressemblance avec le prénom féminin homophone Alice. En effet, si dans le nom Alexandre on trouve le souvenir du nom glorieux d'Alexandre le Grand, le nom Alis peut suggérer une variante trop courte, voire mesquine et caricaturale du premier. On peut facilement se rendre compte qu'Alis n'est que la tentative échouée d'être l'égal de son frère.

Un autre diminutif, rencontré cette fois-ci dans Perceval est Yvonet, nom d'un personnage sans importance, qui accompagne occasionnellement le héros et le soutient par sa bienveillance. Le nom ne suggère guère l'héroïsme, le courage, les grands faits d'armes, mais plutôt laisse s'apercevoir la sympathie accordée à ce personnage qui aide volontiers le protagoniste.

2. On observe dans le cas de quelques noms propres un jeu de sonorités, comme pour Laudine, la dame de Landuc, fille du duc de Landunet. Un autre cas de syllabes réitérées à effet sonore manifeste c'est le nom de celui qui initie Perceval à la chevalerie, qui s'appelle Gorneman de Gorhaut. Son nom rappellerait, selon Philippe Walter, le royaume de Gorre, qui est le nom de l'Autre monde où Méléagant a emmené la reine Guenièvre. Il pourrait aussi évoquer Gorias, un centre initiatique dans les mythes gaéliques (Walter 2003: 44). Les deux ont en commun l'initiation; donc, le nom de celui qui apprend à Perceval le métier des armes et le code chevaleresque s'avère tout à fait motivé.

Guinganbrésil et Gréoréas sont tous les deux les ennemis de Gauvin; à remarquer que tous les trois noms commencent par la lettre $g$, comme pour avoir le même poids. Le mème jeu de sonorité se manifeste dans le nom d'Anguingueron, le sénéchal de Chamadeu qui suggère la violence et la dureté, de même que l'arrogance.

3. Dans la série des noms motivés, qui révèlent le caractère des personnages on peut citer surtout des personnages négatifs, dont le géant Harpin de la Montagne, dont le nom rappelle la harpie, monstre antique; l'Orgueilleuse et l'Orgueilleux de la lande; sire Chamadeu (lier au verbe se chamailler) et d'autres encore.

Les noms peuvent être accompagnés d'une qualité caractéristique de l'individu. Ex.: Uther Pendragon - Uther-à-la-tête de-dragon; Sagremor le Déréglé, appelé ainsi à cause de ses colères subites; le Chevalier Vermeil, dont le nom est donné par la couleur de l'armure. 
Les noms propres peuvent contenir aussi un patronyme, ou une relation de parenté: Erec fils du roi Lac, Enide, fille de Lycorans et de Carsenefide, Yvain, fils d'Urien, Lancelot fils du roi Ban, Gifflès fils de Do, etc.

Les noms peuvent indiquer soit l'origine ou le lieu d'origine, soit la provenance d'un domaine ou territoire possédé: Perceval le Gallois, Lancelot du Lac, (a ne pas confondre avec le roi Lac, père d'Érec), Governal de Roberdic, la dame de Noroison, le seigneur de Noire-Épine, Mélian de Lis, Thibaut de Tintagel, Chamadeu des Iles, l'Orgueilleuse de Nogres, l'Orgueilleux de la Lande, l'Orgueilleux du Passage à l'Étroite Voie, etc.

4. Les objets peuvent porter un nom écrit avec majuscule, et il s'agit en géneral d'un substantif suivi d'un adjectif qualificatif.

Ex.: La Table Ronde, le Lit périlleux.

La Table Ronde est le centre de l'univers arthurien, qui regroupe autour d'elle les chevaliers; selon Jean Frappier, elle est „le centre à la fois géometrique et poétique de toutes les aventures". On y a vu une image réduite du cosmos, suggérant par sa forme ronde la perfection et l'achèvement.

Seulement le Graal ne comporte d'habitude aucun adjectif, sauf celui de saint (le Saint Vase), ayant une signification mystique et sacrée qui ne rend pas possibles d'autres associations.

Chez Chrétien de Troyes, différents noms communs de choses abstraites peuvent recevoir une majuscule, étant ainsi individualisés. C'est le cas des sentiments et des états d'âme - Amour, Honte, mais aussi des principes comme Raison, Nature, Fortune.

Les noms attribués aux situations, aux objets et aux endroits périlleux mettent en valeur la fascination de l'interdit des héros en quête d'aventures. L'idée de danger est visiblement présente dans les appellations Le lit périlleux, le Lit de la Merveille, le Gué périlleux, le Passage à l'Étroite Voie, qui sont des épreuves auxquelles doit se soumettre le héros et dont le nom constitue un avertissement contre les risques éventuels. Le héros sait donc à quoi il a affaire, car la réalité n'y est jamais cachée, ce qui ne l'empêche pas de vouloir mettre à l'épreuve ses forces et d'essayer de dépasser ses limites.

5. Les noms de lieu appartiennent, pour la plupart, à un espace imaginaire et à une carte très floue. Les châteaux par lesquels passent les chevaliers constituent autant de repères et de lieux initiatiques sur leur parcours. Ainsi sont le Château de la Pire Aventure (ou de la Pesme-Aventure), Le Château de Beaurepaire, le chateau des pucelles, le château Orgueilleux, etc.

Les endroits portent des noms qui rendent manifeste leur nature: la Gaste Forêt (stérile, déserte), l'Ile-aux-pucelles, le Pont dessous l'eau, le Pont de l'Épée, le Gué périlleux.

\section{Conclusion}

En analysant les noms des personnages de Chrétien de Troyes nous avons observé les caractéristiques d'une „étymologie ontologique”, car chaque personnage donne la mesure de son nom, le reflète dans sa psychologie et dans ses actions. Cela 
permet au lecteur de mieux s'orienter dans le texte, de repérer des indices importants afin d'accéder à la compréhension du message global du texte, qui s'enrichit ainsi de nouvelles valences.

\section{Bibliographie}

Berthelot, Anne. 1998. Le chevalier à la charrette, Le chevalier au lion, Chrétien de Troyes. Résumé analytique, commentaire critique, documents. Paris: Nathan.

Bromwich, R. 1961. Trioedd Ynys Pridein. The Welsh Triads. Cardiff: inconnu.

Buridant, Claude. 1990. Définition et étymologie de la lexicographie et la lexicologie médiévale. Dans Centre d'études du lexique. La définition, J. Chauraud et F. Mazière (édit.), 43-57. Paris: Larousse.

Buridant, Claude. 1998. Les paramètres de l'étymologie médiévale. Dans L'Étymologie, de l'Antiquité à la Renaissance, C. Buridant (coord.), 11-56. Paris: Presses Universitaires de Septentrion.

Chrétien de Troyes. 2004. Perceval ou le Roman du Graal suivi d'un choix de continuations. Préface d'André Hoog. Paris: Gallimard.

Chrétien de Troyes. 2011. Romans de la Table Ronde. Érec et Énide. Cligès ou la Fausse Morte. Lancelot le Chevalier à la charrette. Yvain le Chevalier au lion. Préface, trad. et notes de J.-P. Foucher. Paris: Gallimard.

Foucher, J.-P. 2011. Préface à Chrétien de Troyes, Romans de la Table Ronde. Érec et Énide. Cligès ou la Fausse Morte. Lancelot le Chevalier à la charrette. Yvain le Chevalier au lion, 7-29. Paris: Gallimard.

Genette, Gérard. 1976. Mimologiques. Voyage en Cratylie. Paris: Seuil.

Greimas, A. J. 1970. Dictionnaire de l'ancien français jusqu'au milieu du XIVe s. Paris: Librairie Larousse.

Kahane, Henry, Renée Kahane. 1961. L'énigme du nom de Cligès. Romania 82 (325): 113-121.

Meuleau, Maurice. 2014. Histoire de la chevalerie. Lille-Rennes: Éditions Ouest-France.

Pavel, Constantin. 2004. Genres et techniques littéraires dans la France médiévale. Iași: Casa Editorială Demiurg.

Sasu, Voichița-Maria. 2006. De l'amour de l'aventure à l'aventure de l'amour. Iași: Casa Editorială Demiurg.

Vişan, Ruxandra. 2003. Échos des pratiques guerrières dans Yvain de Chrétien de Troyes. Dans La chevalerie du Moyen Age à nos jours. Mélanges offerts à Michel Stanesco, Mihaela Voicu și V.-D. Vlădulescu (coord.), 52-68. București: Editura Universității din București.

Walter, Philippe. 2003. Arthur, l'ours-roi et la Grande Ourse. Références mythiques de la chevalerie arthurienne. Dans La chevalerie du Moyen Age à nos jours. Mélanges offerts à Michel Stanesco, Mihaela Voicu și V.-D. Vlădulescu (coord.), 40-51. București: Editura Universității din București.

Zimmer, Heinrich. 1994. Regele și cadavrul. Meditații despre biruința sufletului asupra răului. Traducere de Sorin Mărculescu. București: Humanitas. 